\title{
EXPECTATIVA Y SATISFACCIÓN ESTUDIANTIL POR EL SERVICIO ACADÉMICO DE LA UNIVERSIDAD PRIVADA DE TACNA EN EL AÑO 2015
}

\author{
Expectation and student satisfaction with the academic service of the Private University \\ of Tacna in 2015
}

\section{Omar Alberto Juan Eyzaguirre Reinoso²}

\section{RESUMEN}

Objetivo: El objetivo de la investigación fue determinar la relación existente entre la expectativa y la satisfacción estudiantil por el servicio académico que brinda la Universidad Privada de Tacna durante el desarrollo académico del año 2015.

Método: La presente investigación es de tipo básica. El problema a investigar corresponde a un diseño descriptivo correlacional. La investigación, se llevó a cabo en la Universidad Privada de Tacna en el periodo del año académico 2015. Las unidades de estudio fueron los estudiantes de la Universidad Privada de Tacna. La población estuvo constituida por 5,289 estudiantes matriculados en la Universidad Privada de Tacna en 6 facultades y 20 carreras. La muestra está conformada por 350 estudiantes, tamaño muestral calculado con la tabla de Fisher - Arkin Coltón con un $+-5 \%$ de margen de error. Se trabajó con la encuesta como técnica para establecer los niveles de expectativas y de satisfacción de los estudiantes sujetos del estudio. Los instrumentos para recoger la información fueron: Cuestionario 1 para recoger información sobre el nivel de expectativa de los estudiantes y el cuestionario 2 para la satisfacción.

Resultados: Según las dimensiones que el estudio considera. En ella se puede apreciar que la mayoría de los estudiantes se ubican en una expectativa moderada a excepción de la dimensión práctica pre profesional, donde la mayoría se ubica en la categoría baja expectativa. Sin embargo, es necesario mencionar que las altas expectativas por estas dimensiones están por debajo del $30 \%$ de los estudiantes

\footnotetext{
2 Doctor en educación con mención en Gestión Educativa
}

sujetos del estudio. Respecto de la variable satisfacción estudiantil, se puede señalar que la mayoría de los estudiantes se ubican en la categoría satisfacción moderada. Puntualmente se puede señalar que la infraestructura y servicios universitarios es la que despierta mayor satisfacción, mientras vida universitaria se ubica en el lado opuesto. Sin embargo, es necesario mencionar que la satisfacción en estas dimensiones está por debajo del $23,43 \%$ de los estudiantes sujetos del estudio.

Conclusión: Desarrollada la prueba estadística se ha logrado establecer que existe relación entre la expectativa y la satisfacción estudiantil en estudiantes de la Universidad Privada de Tacna.
Palabras
claves:
Expectativa, satisfacción, calidad, aprendizaje, estudiante universitario

\section{ABSTRAC}

Objective: The objective of the research was to determine the relationship between expectation and student satisfaction with academic service provided by the Private University of Tacna academic development during 2015.

Method: This research is of basic type. The research problem corresponds to a descriptive design - correlational. The research was conducted at the Private University of Tacna in the period of the academic year 2015. Units of study were students of the Private University of Tacna. The population consisted of 5,289 students enrolled in the Private University of Tacna in 6 faculties and 20 runs. The sample consists of 350 students, the calculated sample size table Fisher - Arkin - COLTON $a+-5 \%$ margin of error. We worked with the survey as a technique to establish the levels of expectations and satisfaction of students study subjects. The instruments to collect information were: Questionnaire 1 to 
collect information on the level of expectation of students and 2 for satisfaction questionnaire.

Results: According to the study considers dimensions. It can be seen that most students are placed in a moderate expectation except for professional pre practical dimension, where most are located in the category low expectation. However, it should be mentioned that high expectations for these dimensions are below the $30.00 \%$ of students study subjects. Regarding the variable student satisfaction, it can be noted that most students are placed in the category moderate satisfaction. Punctually it is noted that the university infrastructure and services is aroused greater satisfaction, while university life is located on the opposite side. However, it is necessary to mention that satisfaction in these dimensions is below $23.43 \%$ of students study subjects.

Conclusion: Developed the statistical test has been established that there is a relationship between expectation and student satisfaction Students of the Private University of Tacna.

Keywords: Expectation, satisfaction, quality, learning, college student.

\section{INTRODUCCIÓN}

La universidad, según la nueva ley universitaria $N^{\circ} 30220$, tiene como uno de sus fines "Formar profesionales de alta calidad de manera integral y con pleno sentido de responsabilidad social de acuerdo a las necesidades del país" (Art. 6 inciso 6.2). Para lograr alcanzar esta finalidad, la satisfacción, considerada como una actitud frente una actividad que se realiza, la misma que puede ser positiva o negativa generando satisfacciones e insatisfacciones, ocupa un lugar importante, ya que sólo la persona que se encuentra satisfecha frente a una actividad que recibe podrá alcanzar sus fines en forma exitosa.

La insatisfacción por una actividad, afecta los diferentes componentes de la actividad que se recibe, en el caso que esta sea el servicio académico, afectará: a la formación profesional del estudiante, que evidentemente no será la mejor; en esta actividad la actuación del estudiante se constituye en el eje de la misma, y si no se encuentra satisfecho con la formación que recibe, no podrá prepararse adecuadamente para desempeñar una profesión. Por otro lado, afecta a la imagen de la institución, y por lo tanto al posicionamiento de la misma, en la actividad universitaria. El estudiante insatisfecho, difícilmente se identifica con su institución y no logra una buena comunicación con los demás actores. Esta situación afecta a las posibilidades de alianzas estratégicas, es decir a la posibilidad de firma de convenios con otras instituciones de reconocido prestigio, ya que éstas exigirán que la institución tenga por lo menos un nivel similar al suyo.

La insatisfacción también afecta a los profesionales que egresan y brindan sus servicios a la comunidad. Ya que se sentirán en desventaja con otros profesionales que egresan de universidades donde el grado de satisfacción es bueno. Por esta razón la satisfacción estudiantil, se constituye en un factor de estudio de la presente investigación. La Universidad Privada de Tacna se encuentra preparándose para lograr la acreditación con la CNA de Colombia. Para tal fin se han venido mejorando los servicios que se brindan en ella.

La enseñanza que se brinda en la universidad es de calidad, en la medida que los profesionales que enseñan reúnen las condiciones pedagógicas necesarias, y aplican un sistema de evaluación justo y equitativo. Asimismo, una organización académica ineficaz presentará limitaciones en los contenidos, en la organización del tiempo, en los canales que permitan la comunicación docente-alumnos, alumno-administrativo, en el servicio de tutoría y la organización de la práctica pre profesional. 
El estudio a realizar es pertinente, con la misión y visión de la universidad y con la temática que se aborda relacionada muy de cerca con la mejora continua y la acreditación, tema que por las exigencias de las leyes: Universitaria $\mathrm{N}^{\circ} 30220$ y del SINEACE, que se encuentra en restructuración cobra una actualidad relevante. Cuando el estudiante universitario ingresa a la universidad, ha logrado una meta que es celebrada por padres, familiares y por supuesto el mismo. Sin embargo, pasada la euforia, debe pensar sobre qué es lo que va a hacer en la universidad que espera encontrar en ella, y esto ya es una expectativa.

Según Pichardo y col. (2007), "las investigaciones en este campo (...) pueden agruparse en dos tipos: aquellos que investigan las expectativas del alumnado con la intención de conocer qué esperan de la universidad en general, y aquellos que estudian las expectativas de los estudiantes sobre componentes específicos del proceso de enseñanza-aprendizaje". (p. 4).

Centrado el tema es necesario definir que se entiende por expectativa: Para Carrillo y Ramírez (2011) "las expectativas son posibilidades razonables y sustentadas de que algo suceda, no es cualquier esperanza o aspiración. Se constituyen a partir de experiencias previas, deseos y actitudes".(p. 38). A partir de esta definición, para la presente investigación se considera que la expectativa es la esperanza que el servicio académico de la universidad, sea tal cual él lo desea o percibe hacia un futuro contrastable.

La teoría del aprendizaje social (Bandura. 1987) Esta teoría es citada por Almaguer (2011), y señala: "La sucesión de ciertos fenómenos del medio son regularidades que hacen que se creen expectativas que relacionan a unos con otros, así las personas tienden a prever los sucesos y sus posibles consecuencias, llevándolos a regular su conducta ante las situaciones que se le presentan". (p. 23)

La teoría del aprendizaje social afirma que "Las expectativas se forma en una persona ante una situación determinada, nada está dicho, ya que la conducta esperada de una persona tiene que ver con tan diferentes y variados aspectos como lo son, el momento, el lugar, así como a la persona que se dirige" (Almaguer, 2011, p. 24)

"Para Vroom (1999), la teoría de las expectativas se basa en que el esfuerzo que realizan las personas para obtener un alto desempeño, va a depender de la posibilidad de lograrlo y una vez alcanzado se obtendrá una recompensa" (Carrillo y col, 2011, p. 39)

El estudio que se aborda se enmarca dentro de las dos primeras teorías que se refieren a la esperanza que el deseo que tiene una persona sobre la forma en que se brinda el servicio educativo, pueda cumplirse, pueda hacerse realidad. Bandura (1986), citado por Martínez, y col (2013) considera que "las expectativas de resultados pueden ser de tres tipos: materiales (retribución económica, estabilidad en el empleo, jornada laboral, etc.), sociales (prestigio, poder, influencia, estatus, aprobación paterna, etc.) o personales (satisfacción, autorrealización, etc.). Llevado al terreno de los intereses laborales, estas expectativas de resultados pueden estar afectando a la elección de los estudios y a la satisfacción con los mismos." (p. 3)

La expectativa de los estudiantes se enmarca más en las expectativas sociales y personales, considerando que muchos buscan los estudios universitarios conducentes a una profesión como un vehículo de movilidad social, y otros como una meta personal a ser alcanzada.

La satisfacción como un estado mental del estudiante o una acción por atender una necesidad, está en función a la calidad, en este caso del servicio de formación profesional que se brinda. Por esta razón, en un momento que la actividad universitaria está dirigida a lograr la 
acreditación de la calidad del servicio que brinda, la satisfacción, se constituye en una variable que no se puede dejar de conocer.

Jiménez (2011) citado por Álvarez (2014) considera que: "La satisfacción del estudiante es un elemento clave en la valoración de la calidad de la educación, ya que refleja la eficiencia de los servicios académicos y administrativos: su satisfacción con las unidades de aprendizaje, con las interacciones con su profesor y compañeros de clase, así como con las instalaciones y el equipamiento. La visión del estudiante, producto de sus percepciones, expectativas y necesidades, servirá como indicador para el mejoramiento de la gestión y el desarrollo de los programas académicos. (p. 6)."

En este concepto, se aprecia que se liga estrechamente la satisfacción del servicio académico a la eficiencia del mismo. Sin embargo, se debe señalar que el término satisfacción del estudiante tiene un origen en el campo de la gestión, considera que un alumno satisfecho es un elemento valioso para probar la calidad de una institución educativa, y esto será posible cuando sus necesidades sean atendidas por los servicios de la universidad.

El servicio académico es una actividad que en el mundo universitario es el eje de toda la formación académica, por esta razón se debe tener en cuenta que: La satisfacción del estudiante refleja la eficiencia de los diversos aspectos que componen el día a día de su experiencia educacional, estando está relacionada con el rigor de los cursos, con las interacciones con su profesor y los otros estudiantes, con la justicia con que se le evalúa, etc., así como con las instalaciones y equipamiento que apoyan dichas interacciones (Flores, 2003, p.81)

Es evidente que la satisfacción tiene que ver con la actuación de los actores de la vida universitaria. Es decir que se encuentra relacionada con el estudiante, el desempeño docente, la calidad de gestión y de los servicios que acompañan a la formación del estudiante, etc. La razón de medir la satisfacción de los estudiantes, radica en el hecho de que son ellos el factor principal y garantía de la existencia y mantenimiento de las organizaciones educativas. Los estudiantes son los destinatarios de la educación, son ellos los que mejor pueden valorarla y, aunque tienen una visión parcial, su opinión proporciona un referente que debe tomarse en cuenta. Así en la satisfacción estudiantil el contexto, la vida universitaria vendrían a constituir los factores de higiene y su intención e interés de formarse como profesional serían los factores motivacionales.

Al abordar el tema del servicio académico, se debe desarrollar el tema de la actividad académica. Se cita a Valderrama (s/a) quien manifiesta: "Se ha definido la actividad académica como formada por seis actividades, a las que propongo asignar una ponderación razonable de acuerdo a estándares nacionales e internacionales: docencia, investigación, extensión, perfeccionamiento, administración y generación de recursos (p.6)." Si se tiene en cuenta las actividades planteadas por Valderrama (s/a), se puede considerar las mismas que se plantean cuando se refiere al servicio académico, de allí que es necesario desarrollar cada una de ellas.

La docencia tiene como campo de acción el desarrollo de clases y cursos; sin embargo, se debe reconocer que el objeto de la misma recae también en el educando. Para la atención de este servicio, es imprescindible contar con profesionales que, en la universidad, no necesariamente cuentan con habilidades pedagógicas, pero que desarrollan esta actividad. Es evidente que la actividad académica es un concepto complejo, que puede abordarse desde distintos ángulos: De la autoridad, del docente y del estudiante, por mencionar algunos. Es importante conocer lo que piensa el estudiante de la actividad académica ya que se le considera como el sujeto de la misma, por el que recibe la acción de esta actividad, como una condición básica para su formación profesional. 
El tema en mención amerita abordar una serie aspectos inherentes a él. El servicio es concebido como una actividad dirigida a satisfacer una necesidad de un usuario o cliente. Todo servicio se administra, se brinda de manera planificada y de acuerdo a una estructura establecida, donde se pueden apreciar en el caso del servicio académico, componentes como: Currículo, formación investigativa, académico, evaluación, alumno universitario (estudiante).

Para Lundgren (citado por Lanfancesco, 1992) "El currículum es: a) Una selección de contenidos y fines para la reproducción social, una selección de qué conocimientos y qué destrezas han de ser transmitidos por la educación; b) Una organización del conocimiento y las destrezas; c) Una indicación de métodos relativos a cómo han de enseñarse los contenidos seleccionados. Por lo tanto, el currículum es el conjunto de principios sobre cómo deben seleccionarse, organizarse y transmitirse el conocimiento y las destrezas en la institución escolar". (p.23). Concebido así el currículo, en la universidad, se encuentra en función a un perfil o competencias que debe lograr el profesional que sigue una carrera profesional. Requiere de aprendizajes conceptuales, procedimentales y actitudinales, que el estudiante debe lograr para desempeñarse como profesional dentro de una comunidad.

Según Vargas (2010) considera que "la investigación como la innovación son estrategias para la calidad de la educación y uno de sus aportes fundamentales está en la construcción del maestro $y$, en general, de los actores educativos como sujetos pedagógicos." (p.1). Definitivamente, la investigación es una estrategia de formación profesional, de allí que se encuentra íntimamente ligada al currículo y a la actividad del docente.

Según Jara (1997) "Los académicos (...) son un cuerpo de creadores-formadores que son capaces de producir y transmitir conocimiento como parte de un proceso liberador de entendimiento de nuestro devenir. Individualmente, demuestran su vocación, su compromiso ético y su decisión libre de realizar su proyecto de vida desde la institución. (...) En su desarrollo, el académico, debe llegar a mostrar autonomía en el sentido de hacer lo que él es, y de ser reconocido por el resultado en creación y formación." (p.4).

El servicio académico, requiere de un componente que conduzca, oriente, facilite el proceso de aprendizaje en la formación profesional, en función al currículo y al manejo de estrategias de aprendizaje. Además, debe ser capaz de planificar y valorar en forma efectiva todo proceso de formación. Por lo tanto, Jara no se equivoca cuando considera al docente como un creador capaz de producir y trasmitir conocimientos, con compromiso ético, identificado con la institución.

Tapia (1985) considera que la "evaluación, es el acto por el cual, el responsable de la actividad que desempeña, juzga en función de determinados indicadores los resultados a que llegan, otorgándoles una estimación, valor y apreciación que le va a permitir tomar decisiones posteriores" (Citado por Valdivia, 2001, p. 55). Si esta definición se traslada al campo educativo, el responsable de la actividad es el docente y que con indicadores, deberá medir el resultado final del proceso enseñanza aprendizaje, y decidir el nivel alcanzado por el estudiante.

Es evidente que si bien el aprendizaje está a cargo del estudiante y que de acuerdo al paradigma actual él mismo genera sus aprendizajes, estos deben ser evaluados, ya que al final de acuerdo al currículo, todo aprendizaje es planificado en la formación profesional. Por lo tanto, se debe verificar el cumplimiento de objetivos y el logro de las competencias. Tapia (1985) describe muy bien, en que consiste esta actividad de verificar, de evaluar, de juzgar el nivel alcanzado por el estudiante al término del proceso de aprendizaje y señalar si se encuentra en condiciones de continuar su formación profesional. 
Este componente es considerado, dentro del modelo pedagógico, como el eje de todo proceso enseñanza aprendizaje. Fernández y otros (2010) señalan que: "El alumno no se puede considerar como un mero comprador o receptor pasivo de un producto; es necesario considerar su participación en el proceso de enseñanza/aprendizaje. El énfasis en el alumno y en satisfacer sus necesidades puede parecer un planteamiento arriesgado si, como señalan algunos autores (...), el estudiante no sabe lo que quiere cuando entra en la Universidad, llegando incluso a considerar denigrante este objetivo. (p.18)." Son los estudiantes los principales usuarios de los servicios universitarios, los destinatarios de la educación, son ellos los que mejor pueden valorarla, $y$, aunque pueden tener una visión parcial, sus opiniones no dejan de ser fruto de sus percepciones, influenciadas por expectativas, necesidades y por diversos factores, que sirven como indicador de mejoramiento de la gestión y el desarrollo de los programas académicos. (Jiménez, et al. 2011, p. 46)

El estudiante universitario, es el centro, el eje de todo proceso de formación profesional. Requiere de aprender conocimientos, dominar habilidades que le demande su profesión y formarse en los valores de la deontología de su carrera. El estudiante no es que no sepa lo que quiere cuando entra a la universidad como dice Barret (1996) citado por Fernández (p.18). El ha ingresado a la universidad porque quiere ser profesional y frente a ese deseo, se le debe orientar. El estudiante tiene expectativas y necesidades que atender. Tiene una esperanza de lograr en la universidad lo que el viene buscando y demostrar o no su satisfacción.

\section{MATERIALES Y MÉTODO}

La presente investigación es básica, con un diseño descriptivo - correlacional. El ámbito de la investigación es Microregional, se llevó a cabo en la Universidad Privada de Tacna en el periodo del año académico 2015. Las unidades de estudio fueron los estudiantes de la Universidad Privada de Tacna. La población estuvo constituida por 5,289 estudiantes matriculados en la Universidad Privada de Tacna en 6 facultades y 20 carreras. La muestra está conformada por 350 estudiantes, tamaño muestral calculado con la tabla de Fisher - Arkin - Coltón con un + - 5\% de margen de error. La recolección de datos se hizo personalmente y de primera fuente recogiendo la información sobre las expectativas y satisfacción estudiantil de los estudiantes de la UPT. Se trabajó con la encuesta como técnica para establecer los niveles de expectativas y de satisfacción de los estudiantes sujetos del estudio. Los instrumentos para recoger la información fueron: Cuestionario 1 para recoger información sobre el nivel de expectativa de los estudiantes y el cuestionario 2 para la satisfacción.

\section{RESULTADOS}

La confiabilidad de los instrumentos se realizó mediante el coeficiente Kuder-Richardson obteniendo un resultado de 0.94 para la expectativa y 0.93 para la satisfacción lo que indica que tiene una alta confiabilidad. La validez del instrumento se efectuó mediante el juicio de expertos alcanzando una aprobación de $92.2 \%$.

Análisis estadístico sobre el nivel de expectativa por el servicio académico que presentaron los estudiantes de la Universidad Privada de Tacna al iniciar el desarrollo académico del 2015

Para establecer el nivel de expectativa por el servicio académico que presentaron los estudiantes, se ha trabajado con cinco dimensiones, y los resultados son los siguientes: 
Tabla 1. Comportamiento de los niveles de expectativa de los estudiantes por el Servicio Académico que brinda la Universidad Privada de Tacna

\begin{tabular}{lccc}
\hline Dimensiones de la expectativa & Alta & Moderada & Baja \\
\hline Gestión Universitaria & 103 & 177 & 70 \\
Actividad académica & 91 & 165 & 94 \\
Actividad administrativa & 88 & 149 & 113 \\
Bienestar estudiantil & 46 & 155 & 149 \\
Práctica profesional & 73 & 131 & 146 \\
\hline
\end{tabular}

Fuente: Tablas estadísticas

\section{INTERPRETACIÓN}

En la Tabla 1 se presenta la información sobre el comportamiento de la expectativa que presentan los estudiantes de la Universidad Privada de Tacna al inicio del año académico 2015, según las dimensiones que el estudio considera. En ella se puede apreciar que la mayoría de los estudiantes se ubican en una expectativa moderada a excepción de la dimensión práctica pre profesional, donde la mayoría se ubica en la categoría baja expectativa. Específicamente se puede señalar que la gestión universitaria es la que despierta mayor expectativa, mientras el bienestar estudiantil se ubica en el lado opuesto. Sin embargo, es necesario mencionar que las altas expectativas por estas dimensiones están por debajo del $30 \%$ de los estudiantes sujetos del estudio.

De otro lado, se aprecia que el comportamiento del nivel moderado tiende una tendencia a la baja. El punto mayor le corresponde a gestión universitaria, y el punto más bajo a la práctica profesional. El nivel moderado de la expectativa estudiantil se encuentra entre el $50.57 \%$ y el $37.43 \%$.

En cuanto al nivel de baja expectativa, se aprecia un comportamiento ascendente. La mayor cantidad de estudiantes le corresponde al bienestar estudiantil y la menor cantidad de ellos en gestión universitaria. Este nivel se encuentra ubicado entre el $42.57 \%$ y el $20.00 \%$, de las unidades de la investigación.

Por lo tanto se puede señalar que la expectativa estudiantil en su mayoría es aceptable, pero no la mejor.

\section{Análisis estadístico sobre el nivel de satisfacción por el servicio académico que presentaron los estudiantes de la Universidad Privada de Tacna al finalizar el desarrollo académico del año 2015.}

Para trabajar el nivel de satisfacción por el servicio académico que presentaron los estudiantes, se ha trabajado con cuatro dimensiones, y sus indicadores. Estas dimensiones son las siguientes:

Tabla 2. Comportamiento de los niveles de satisfacción de los estudiantes por el Servicio Académico que brinda la Universidad Privada de Tacna

\begin{tabular}{lccc}
\hline Dimensiones de la satisfacción & Alta & Moderada & Baja \\
\hline Enseñanza & 76 & 204 & 70 \\
Organización académica & 61 & 183 & 106 \\
Vida Universitaria & 70 & 173 & 107 \\
Infraestructura y servicios universitarios & 82 & 158 & 110 \\
\hline
\end{tabular}

Fuente: Tablas estadísticas 


\section{INTERPRETACIÓN}

En la Tabla 2 se presenta la información sobre el comportamiento de la satisfacción que presentan los estudiantes de la Universidad Privada de Tacna al finalizar el año académico 2015, según las dimensiones que se consideran en el estudio. Allí se puede apreciar que la mayoría de los estudiantes se ubican en la categoría satisfacción moderada.

Puntualmente se puede señalar que la infraestructura y servicios universitarios es la que despierta mayor satisfacción, mientras vida universitaria se ubica en el lado opuesto. Sin embargo, es necesario mencionar que la satisfacción en estas dimensiones está por debajo del $23,43 \%$ de los estudiantes sujetos del estudio.

Asimismo, se aprecia que el comportamiento del nivel moderado tiene una tendencia a la baja. El mayor valor le corresponde a la enseñanza, y el valor más bajo a la infraestructura. El nivel moderado de la satisfacción estudiantil se encuentra entre el $58,29 \%$ y el $45,14 \%$.

Finalmente, en el nivel de baja satisfacción, se aprecia un comportamiento ascendente. La mayor cantidad de estudiantes insatisfechos le corresponde a la infraestructura y servicios universitarios y la menor cantidad de ellos se ubica en la enseñanza. Este nivel se encuentra ubicado entre el $31,43 \%$ y el $20,00 \%$, de las unidades de la investigación.

Por lo tanto, se puede señalar que si bien la satisfacción estudiantil en su mayoría es aceptable, no necesariamente es lo mejor, en la prestación de un servicio académico.

\section{Relación estadística entre la expectativa y la satisfacción estudiantil por el servicio académico que brinda la Universidad Privada de Tacna durante el desarrollo académico del año 2015.}

La relación entre las variables del estudio se trabaja con la prueba de Chi cuadrado. Para tal fin se ha considerado una probabilidad del 0,05; los grados de libertad son 4 (gl); y un valor crítico de 9,488.

\section{PRUEBA DE CHI CUADRADO}

HIPÓTESIS ESTADÍSTICAS

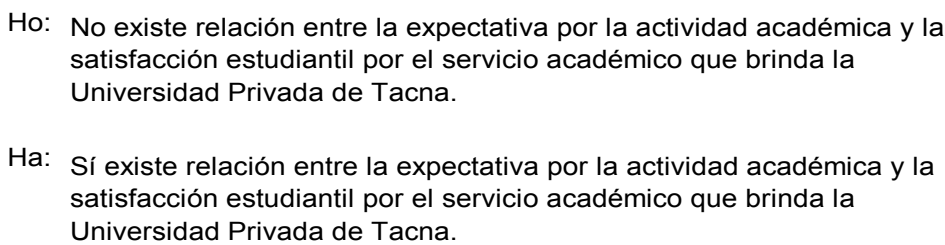

DESARROLLO

\begin{tabular}{|l|c|c|c|r|}
\hline $\begin{array}{c}\text { Expectativapor la actividad } \\
\text { académica }\end{array}$ & Alta & Moderada & Baja & \multirow{2}{*}{ TOTAL } \\
\cline { 2 - 4 } & 45 & 45 & 1 & $\mathbf{9 1}$ \\
\hline Alta expectativa & 30 & 89 & 46 & $\mathbf{1 6 5}$ \\
\hline Expectativa moderada & 1 & 40 & 53 & $\mathbf{9 4}$ \\
\hline Baja expectativa & $\mathbf{7 6}$ & $\mathbf{1 7 4}$ & $\mathbf{1 0 0}$ & $\mathbf{3 5 0}$ \\
\hline TOTAL & \multicolumn{2}{|r}{}
\end{tabular}

Tabla de contingencia

Tabla de contingencia
\begin{tabular}{|r|r|r|r|r|r|}
\hline Celdas & fo & fe & fo $-\mathrm{fe}$ & (fo - fe) 2 & (fo - fe) $2 / \mathrm{fe}$ \\
\hline 1 & 45 & 19,8 & 25,2 & 637,1 & 32,2 \\
\hline 2 & 30 & 35,8 & $-5,8$ & 34,0 & 0,9 \\
\hline 3 & 1 & 20,4 & $-19,4$ & 376,8 & 18,5 \\
\hline 4 & 45 & 45,2 & $-0,2$ & 0,1 & 0,0 \\
\hline 5 & 89 & 82,0 & 7,0 & 48,6 & 0,6 \\
\hline 6 & 40 & 46,7 & $-6,7$ & 45,3 & 1,0 \\
\hline 7 & 1 & 26,0 & $-25,0$ & 625,0 & 24,0 \\
\hline 8 & 46 & 47,1 & $-1,1$ & 1,3 & 0,0 \\
\hline TOTAL & 53 & 26,9 & 26,1 & 683,4 & 25,4 \\
\hline
\end{tabular}

Probabilidad

gl

Valor crítico

Chi cuadrado 


\section{INTERPRETACIÓN}

Desarrollada la prueba estadística se ha logrado establecer que existe relación entre la expectativa y la satisfacción estudiantil en estudiantes de la Universidad Privada de Tacna.

Considerando que el valor de Chi cuadrado es 189,094, y mayor al valor crítico 9,488 se puede afirmar que se acepta la hipótesis alterna y se rechaza la hipótesis nula, por lo tanto, existe una relación directa y significativa entre la expectativa y la satisfacción que tienen los estudiantes sobre el servicio académico que presta la Universidad Privada de Tacna.

\section{CONCLUSIONES}

a) Los estudiantes de la Universidad Privada de Tacna al inicio el desarrollo académico del 2015, presentaban un nivel moderado en su expectativa del desarrollo del servicio académico. Se destaca la expectativa que tiene por la gestión universitaria y la actividad académica.

b) Los estudiantes de la Universidad Privada de Tacna al finalizar el desarrollo académico del año 2015, presentaron un nivel de satisfacción moderado, por el servicio académico recibido. Destacan las dimensiones Enseñanza y organización académica.

c) Existe una relación directa y muy significativa entre la expectativa y la satisfacción estudiantil por el servicio académico que brindó la Universidad Privada de Tacna durante el desarrollo académico del año 2015. La prueba del Chí cuadrado lo demuestra así.

d) La relación existente entre la expectativa y la satisfacción estudiantil por el servicio académico que brinda la Universidad Privada de Tacna durante el desarrollo académico del año 2015, es intensa. El comportamiento de los componentes (dimensiones) de la expectativa en el impacto en la satisfacción estudiantil, es heterogénea donde destaca la influencia de la actividad administrativa, bienestar estudiantil y la práctica pre profesional.

\section{REFERENCIAS BIBLIOGRÁFICAS}

- Almaguer, M. (2011) Nivel de expectativas de logro profesional en estudiantes de psicología educativa de la UPN, Unidad 095 Azcapotzalco a partir de la opinión de la formación académica (Tesis). México: Universidad Pedagógica Nacional.

- Álvarez, P. et. al. (2014) Estudios de la Satisfacción de los Estudiantes con los Servicios Educativos brindados por Instituciones de Educación

- Carrillo, A. y Ramírez,J. (2011) Expectativas académicas y laborales de estudiantes próximos a egresar de una licenciatura de Psicología Educativa (Tesis) México: Universidad Pedagógica Nacional.

- Caballero, A. (2004) Guías metodológicas para los planes y tesis de maestría y doctorado. Lima Perú: Alen Caro.

- Cavalcante,J. (2004) Satisfacción en el trabajo de los directores de Escuelas Secundarias Públicas de Bahía-Brasil. Tesis) España: Universidad de Barcelona.

- Espinoza (2011) Clima social familiar de parejas con 15 años o más de unión matrimonial del distrito de San Sebastian. Cusco.

- Fernández. et.al. (2010). Calidad Universitaria: expectativas de los estudiantes recién incorporados. Publicado en revista Nacional de Administración. La Rioja.

- Jara,S. (1997) La actividad académica: Preguntas, discusión y Síntesis. Chile.

- Flores,J. (2003) La Satisfacción estudiantil como indicador de la calidad de la educación superior publicado en Revistas investigación de la Universidad Mayor de San Marcos. Lima. 
- Jiménez, A, Terriquez,B y Robles,F(2011) Evaluación de la satisfacción académica de los estudiantes de la Universidad Autónoma de Nayarit. En Revista Fuente Año $3 \mathrm{~N}^{\circ} 6$ Enero - Marzo.

- Jiménez, N. (2004) La formación de las expectativas profesionales de los jóvenes en los procesos de inserción social y profesional Superior del Valle de Toluca. Publicado en la revista Iberoamericana de Calidad, Eficacia y Cambio en Educación, 13 (2) 5-26.

- La Francesco, G.(2003) Nuevos Fundamentos para la transformación curricular. Colombia: Magisterio

- Mejías, A y Martínez,D (2009) Desarrollo de un Instrumento para Medir la Satisfacción Estudiantil en Educación Superior, en Revista Docencia Universitaria Vol- X, No 2. Venezuela: SADPRO UCV.

- Muñoz,M. (2012) Modelo de asociación entre factores de Satisfacción estudiantil y lealtad universitaria validado en alumnos graduados de la Universidad adventista de habla hispana de la división interamericana ( Tesis) México: Universidad de Montemorelos.

- Pichardo, M. et.al. (2007) El estudio de las expectativas en la universidad: análisis de trabajos empíricos y futuras líneas de investigación. Publicado en la Revista Electrónica de Investigación Educativa Vol. $9 \mathrm{~N}^{\circ} 1$.

- Ritchey, F. (2008) Estadística para las ciencias sociales. México: McGraw Hill

- Rodriguez,P y Chávez, (2009) Factores institucionales asociados a la satisfacción del estudiante de Arquitectura de la Universidad Tecnológica de El Salvador. El Salvador: Universidad Tecnológica.

- Sierra, R. (2003) Tesis Doctorales y trabajos de investigación científica. Madrid: Thomson.

- Valderrama, J. (s/a) Un Modelo para la Distribución Racional de la Actividad Académica en una Universidad. Chile: Universidad de la Serena.

- Valdivia, R. (2009) Elaborando la Tesis: una propuesta. Tacna. UPT- Perú

Recibido: 20/03/2016

Aceptado para la publicación

$12 / 4 / 2016$ 